\title{
Probability backflow and a new dimensionless quantum number
}

\author{
A J Bracken and G F Melloy \\ Department of Mathematics, The University of Queensland, Brisbane, Queensland 4072, \\ Australia
}

Received 11 November 1993

\begin{abstract}
Pure states of a free particle in non-relativistic quantum mechanics are described, in which the probability of finding the particle to have a negative $x$-coordinate increases over an arbitrarily long, but finite, time interval, even though the $x$-component of the particle's velocity is certainly positive throughout that time interval. It is shown that, for any state of this type, the greatest amount of probability which can flow back from positive to negative $x$-values in this counter-intuitive way, over any given time interval, is equal to the largest eigenvalue of a certain Hermitian operator, and it is estimated numerically to have a value near 0.04 . This value is not only independent of the length of the time interval and the mass of the particle, but is also independent of the value of Planck's constant. It reflects the structure of Schrödinger's equation, rather than the values of the parameters appearing there. Backflow of positive probability is related to the non-positivity of Wigner's density function, and can be regarded as arising from a flow of negative probability in the same direction as the velocity. Generalizations are indicated, to the relativistic free electron, and to non-relativistic cases in which probability backflow occurs even in opposition to an arbitrarily strong constant force.
\end{abstract}

\section{Introduction}

Imagine a classical particle moving along the $x$-axis, and suppose that at some initial time its position $x$ and its velocity $v(=\mathrm{d} x / \mathrm{d} t)$ are uncertain, whereas it is certain that $v$ is positive. If the particle is subject to no forces, it will subsequently travel in the positive $x$-direction, and it is intuitively obvious that the probability of finding it in the spatial interval $\left(-\infty, x_{0}\right)$ will be a non-increasing function of time, for any given $x_{0}$, say $x_{0}=0$.

In contrast, there exist pure states of a free particle in (non-relativistic) quantum mechanics for which the probability of finding the particle in $(-\infty, 0)$ increases with time for an arbitrarily long (but finite) period, even though at all times the probability equals unity that the particle has positive $x$-component of velocity.

The reader's immediate response may be that there is no great surprise here. When the velocity of the particle is assumed equal to its momentum divided by its mass, then the indeterminacy principle precludes the simultaneous measurement of the position and velocity, and even the position and the direction of the velocity are incompatible observables. However, in the states described, the direction of the velocity (momentum) is determinate and does not require measurement after state preparation, and the increase in the probability of finding the particle in $(-\infty, 0)$, in the absence of any measurements at all, can be confirmed by making position measurements at various times on a large ensemble of similar particles, all prepared in the same state.

It has been noted by students of the 'pilot wave' interpretation of the wavefunction that, in the quantum mechanics of a single particle, the direction of the probability flux vector at 
any particular instant does not necessarily predict the direction of the particle's momentum, measured immediately afterwards (de Broglie 1926, 1960, Bohm 1952, Belinfante 1973), but in these studies, the phenomenon of 'probability backflow' does not seem to have been identified. For the case of a particle moving on the $x$-axis, another velocity

$$
V(x, t)=\frac{j(x, t)}{\rho(x, t)}
$$

is associated with the pilot wave, where $j(x, t)$ is the (x-component of the) probability flux vector and $\rho(x, t)$ is the probability density, as in (8), (9) below. Evidently $V(x, t)$ always has the same sign as $j(x, t)$, but it is very different in form and structure from the velocity operator

$$
v_{\text {op }}=p_{\text {op }} / m \quad p_{\text {op }}=-\mathrm{i} \hbar \partial / \partial x
$$

and it is $v_{\text {op }}$ rather than $V(x, t)$ which represents the observable velocity, according to the standard interpretation of quantum mechanics. The first explicit identification of probability backflow seems to have been made in studies of the arrival-time problem in quantum mechanics by Allcock (1969), who constructed an example to illustrate the effect, although only in terms of an approximate solution to Schrödinger's equation. However, the implications and quantification of such a backflow over a finite time interval do not appear to have been explored in the literature.

It will be argued below that this counter-intuitive quantum effect, in which positive probability apparently flows in the 'wrong' direction, can be regarded as arising from a flow of negative probability in the 'right' direction. The idea that a formal introduction of negative probabilities, in the intermediate stages of calculations, might make some of the peculiarities of quantum mechanics easier to describe, if not to digest, has been discussed by Feynman (Feynman 1987; see also Dirac (1942), Bartlett (1945)). Although Feynman emphasized that he did not 'claim that quantum mechanics is best understood by going back to classical mechanical concepts and allowing negative probabilities', their introduction does provide a strikingly simple interpretation of the peculiar effect under discussion here.

\section{Probability flux vectors}

At the mathematical level, the effect has its origins in the form taken by the probability flux vector in quantum mechanics.

Classically, if $\mu(x, v) \delta x \delta v$ is the probability that at time $t=0$ the free particle is within $\delta x$ of $x$, with velocity within $\delta v$ of $v$, then the corresponding probability at $t>0$ is $\mu(x-v t, v) \delta x \delta v$, and the (x-component of the) probability flux vector at $(x, t)$ is given by

$$
j(x, t)=\int_{0}^{\infty} \mu(x-v t, v) v \mathrm{~d} v .
$$

The integral is restricted to positive values of $v$ because $\mu$ vanishes when $v$ is negative, by assumption in the present case. Because $\mu$ is non-negative, it follows from (3) that $j(x, t)$ is also non-negative, for all values of $x$, and at all positive times.

The probability that the particle is within $\delta x$ of $x$ at time $t$ is $\rho(x, t) \delta x$, where

$$
\rho(x, t)=\int_{0}^{\infty} \mu(x-v t, v) \mathrm{d} v
$$


and the probability that the particle is in the spatial interval $(-\infty, 0)$ is $P(t)$, where

$$
P(t)=\int_{-\infty}^{0} \rho(x, t) \mathrm{d} x
$$

It follows directly from (3) and (4) that $\rho$ and $j$ satisfy the conservation equation

$$
\frac{\partial \rho(x, t)}{\partial t}+\frac{\partial j(x, t)}{\partial x}=0
$$

and it then follows from (5), provided $j(-\infty, t)=0$, that

$$
\frac{\mathrm{d} P(t)}{\mathrm{d} t}=-j(0, t)
$$

Because $j(0, t) \geqslant 0$ for all $t>0$, equation (7) then expresses the result expected intuitively: the probability that the particle is in the spatial interval $(-\infty, 0)$ is non-increasing with time.

In quantum mechanics, a free particle with mass $m$ moving on the $x$-axis is described, when in a pure state, by a normalized wavefunction $\psi(x, t)$. (The following discussion could be formulated using normalized wavefunctions in three-dimensional space, but this would only complicate the arguments unnecessarily.) In terms of $\psi$, a probability density $\rho(x, t)$ and flux vector $j(x, t)$ are defined by

$$
\begin{aligned}
& \rho(x, t)=\psi^{*}(x, t) \psi(x, t) \\
& j(x, t)=-\mathrm{i} \frac{\hbar}{2 m}\left[\psi^{*}(x, t) \frac{\partial \psi(x, t)}{\partial x}-\frac{\partial \psi^{*}(x, t)}{\partial x} \psi(x, t)\right] .
\end{aligned}
$$

Like their classical counterparts, these satisfy the conservation equation (6), in this case as a result of Schrödinger's equation

$$
\mathrm{i} \hbar \frac{\partial \psi(x, t)}{\partial t}=-\frac{\hbar^{2}}{2 m} \frac{\partial^{2} \psi(x, t)}{\partial x^{2}} .
$$

Consequently, if $P(t)$ is defined as in (5), with $\rho$ as in (8), then (7) is again valid, with $j$ as in (9). The critical difference from the classical case is that $j(0, t)$ in (7) can now be negative over some finite time interval, even though the probability equals unity that the velocity of the particle is positive throughout that interval.

The latter is taken to be a consequence of the assumption that the Fourier decomposition of $\psi$ involves only positive momenta at all positive times; only those solutions of (10) will be considered which have the form

$$
\psi(x, t)=\frac{1}{\sqrt{2 \pi \hbar}} \int_{0}^{\infty} \mathrm{e}^{\mathrm{i} x p / \hbar} \mathrm{e}^{-\mathrm{i} p^{2} t / 2 m \hbar} \phi(p) \mathrm{d} p .
$$

The normalization condition on the wavefunction $\psi$ requires that

$$
\int_{0}^{\infty} \phi^{*}(p) \phi(p) \mathrm{d} p=1
$$

By the Born interpretation, this also expresses the fact that the probability equals unity of obtaining a positive value on measurement of the momentum, and hence of the velocity, at any positive time. 
Note that if the expectation values $\langle x\rangle(t)$ and $\left\langle v_{\mathrm{op}}\right\rangle(t)$ are introduced in the usual way, whenever $\psi$ in (11) is such that they are well defined, with

$$
\begin{aligned}
& \langle x\rangle(t)=\int_{-\infty}^{\infty} x \psi^{*}(x, t) \psi(x, t) \mathrm{d} x \\
& \left\langle v_{\mathrm{op}}\right\rangle(t)=\int_{-\infty}^{\infty} \psi^{*}(x, t) v_{\mathrm{op}} \psi(x, t) \mathrm{d} x=\frac{1}{m} \int_{0}^{\infty} p \phi^{*}(p) \phi(p) \mathrm{d} p
\end{aligned}
$$

then $\left\langle v_{\mathrm{op}}\right\rangle(t)=\left\langle v_{\mathrm{op}}\right\rangle(0)>0$, and $\langle x\rangle(t)=\langle x\rangle(0)+t\left\langle v_{\mathrm{op}}\right\rangle(0)$, which increases monotonically with $t$ for all $t>0$.

Note also that if $j(0,0)$ is negative, then $j(0, t)$ will be negative over some time interval $[0, T)$, with $T>0$, by continuity in $t$. However, the consequent flow of probability in the negative $x$-direction over this time interval is not in itself surprising, even if $\psi(x, 0)$ contains only positive momentum components, unless it is also true that $\psi(x, t)$ contains only positive components throughout the interval. In the case of a free particle, as is shown by (11), $\psi(x, t)$ contains only positive components at all positive times, only if it does so at $t=0$.

In order to confirm the existence for a free particle of the surprising quantum effect under discussion, it is therefore only necessary to find a function $\phi(p)$ which vanishes for $p<0$, satisfies (12), and defines through (11), (9) at $t=0$ a flux vector satisfying $j(0,0)<0$.

\section{An illustrative example}

Before proceeding to a realistic example, it is instructive to consider a superposition of two plane waves with positive momenta,

$$
\begin{aligned}
& \psi(x, t)=A \mathrm{e}^{\mathrm{i} \theta_{1}(x, t)}+B \mathrm{e}^{\mathrm{i} \theta_{2}(x, t)} \\
& \theta_{n}(x, t)=\left[p_{n}\left(x-\frac{\left(p_{n} / 2 m\right) t}{\hbar}\right)+\gamma_{n}\right] \quad n=1,2 .
\end{aligned}
$$

Here $A, B, p_{1}$ and $p_{2}$ are positive constants, and the constant phases $\gamma_{1}$ and $\gamma_{2}$ are arbitrary. For a single plane wave with positive momentum, the probability flux vector (9) is a positive constant, but for the superposition (14) it takes the form

$$
j(x, t)=\frac{1}{m}\left(p_{1} A^{2}+p_{2} B^{2}+\left(p_{1}+p_{2}\right) A B \cos \left[\theta_{1}(x, t)-\theta_{2}(x, t)\right]\right)
$$

which at any value of $x$, varies with time between an upper value of $\left(p_{1} A+p_{2} B\right)(A+B) / m$ and a lower value of $\left(p_{1} A-p_{2} B\right)(A-B) / m$. If, for example, $A>B$ and $p_{1} A<p_{2} B$, then this lower value is negative.

Because $\psi$ in (14) is not normalizable, this result has no direct physical interpretation, but it does indicate that the occurrence of negative $j$ values, for a superposition of plane waves with positive momenta, can be regarded as an interference effect. The following example shows that the effect can occur for normalizable wavefunctions.

In (11), set

$$
\phi(p)= \begin{cases}\frac{18}{\sqrt{35 K}} p\left(\mathrm{e}^{-p / K}-\frac{1}{6} \mathrm{e}^{-p / 2 K}\right) & p>0 \\ 0 & p<0\end{cases}
$$




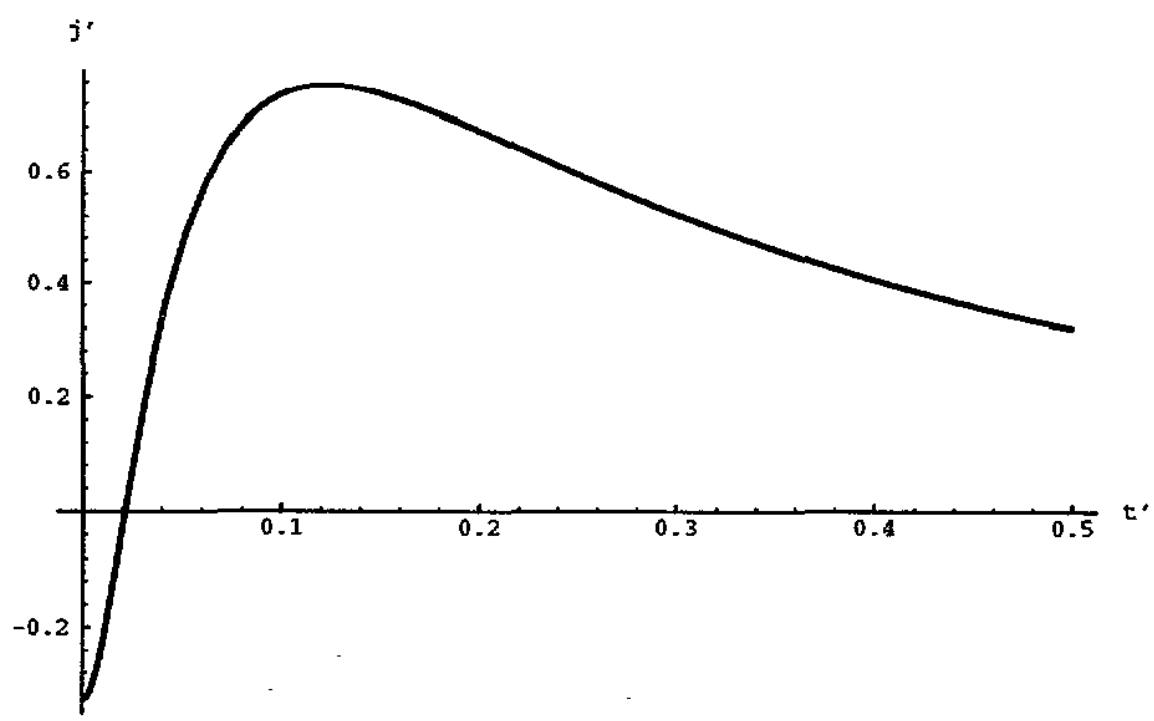

Figure 1. Plot of $j^{\prime}\left(0, t^{\prime}\right)$ against $t^{\prime}$, where $j^{\prime}=m \hbar j / K^{2}, t^{t}=K^{2} t / m \hbar$, showing a time interval over which probability backflow occurs.

where $K$ is a positive constant with dimensions of momentum, and note that (12) is then satisfied. It now follows from (11) that

$$
\psi(x, 0)=18 \sqrt{\frac{K \hbar^{3}}{70 \pi}}\left[\frac{1}{(\hbar-\mathrm{i} K x)^{2}}-\frac{2}{3(\hbar-2 \mathrm{i} K x)^{2}}\right] .
$$

Hence

$$
\psi(0,0)=6 \sqrt{\frac{K}{70 \pi \hbar}} \quad \frac{\partial \psi}{\partial x}(0,0)=-12 \mathrm{i} \sqrt{\frac{K^{3}}{70 \pi \hbar^{3}}}
$$

and

$$
j(0,0)=-\frac{36 K^{2}}{35 \pi m \hbar}<0 .
$$

It also follows from (11) that, for $t>0$ in this example,

$$
\begin{aligned}
& \psi(x, t)=-18 \sqrt{\frac{K}{70 \pi \hbar}}\left(\frac{5 i}{6 t^{\prime}}+\sqrt{\frac{\pi}{4\left(t^{\prime}\right)^{3}}}(1-\mathrm{i})\left\{\left(x^{\prime}+\mathrm{i}\right) \mathrm{e}^{\mathrm{i} f\left(x^{\prime}\right)^{2}+2 \mathrm{ix} x^{\prime}-1 \mathrm{~J} / 2 t^{\prime}} \operatorname{erf}\left[\frac{-(1+\mathrm{i})\left(x^{\prime}+\mathrm{i}\right)}{\sqrt{4 t^{\prime}}}\right]\right.\right. \\
& \left.\left.-\frac{1}{12}\left(2 x^{\prime}+\mathrm{i}\right) \mathrm{e}^{\left.\mathrm{i}\left(x^{\prime}\right)^{2}+\mathrm{ix} x^{\prime}-1 / 4\right) / 2 t^{\prime}} \text { erf }\left[\frac{-(1+\mathrm{i})\left(2 x^{\prime}+\mathrm{i}\right)}{\sqrt{16 t^{\prime}}}\right]\right\}\right)
\end{aligned}
$$

where $t^{\prime}=K^{2} t / m \hbar, x^{\prime}=K x / \hbar$, and erf is the error function. It is then easy to show, with the help of the computer software package Mathematica (1991), that in fact $j(0, t)<0$ from $t=0$ to $t=t_{1} \approx 0.021 \mathrm{mh} / \mathrm{K}^{2}$ in this case, so that $\mathrm{d} P / \mathrm{d} t>0$ over this time interval (see figure 1). According to (5), the increase $\Delta_{P}$ in the probability mass on the spatial interval $(-\infty, 0)$ during this period is equal to the area in figure 1 bounded by the plot of $j^{\prime}\left(0, t^{\prime}\right)$ and the two coordinate axes, which equals 0.0043 , approximately. Thus

$$
\Delta_{P}=P\left(t_{1}\right)-P(0) \approx 0.0043 \text {. }
$$




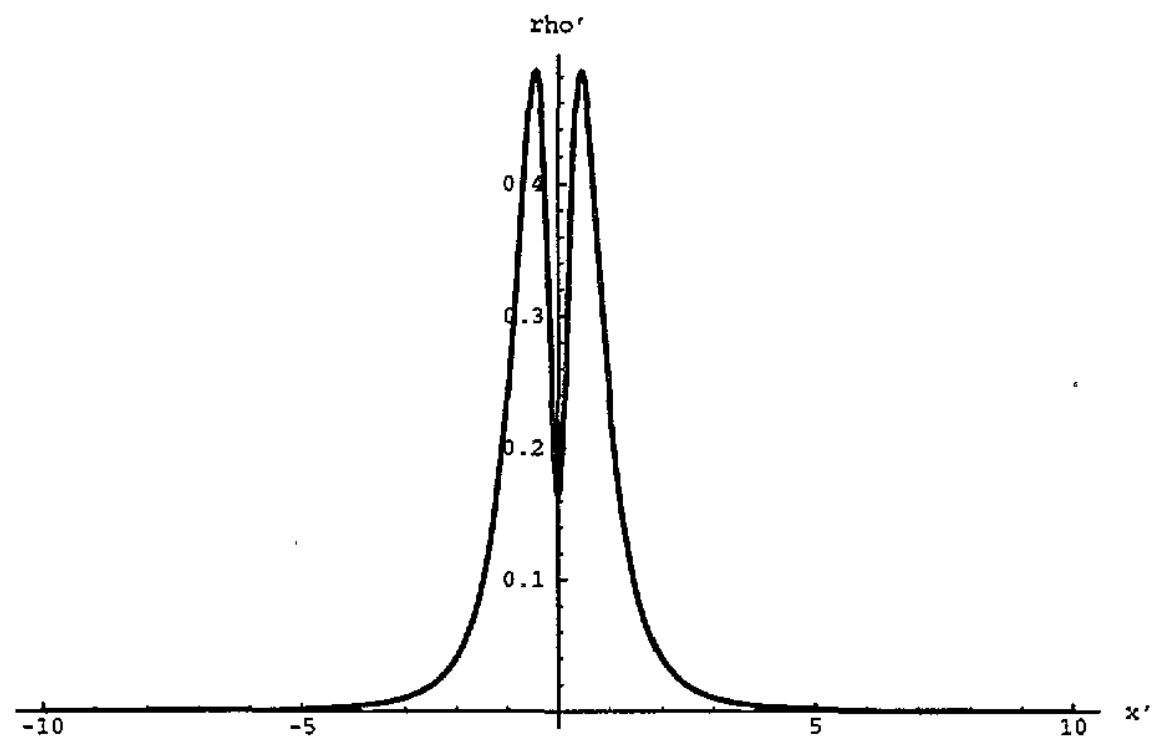

Figure 2. Plot of the even function $\rho^{\prime}\left(x^{\prime}, 0\right)$ against $x^{\prime}$, where $\rho^{\prime}=\hbar \rho / K, x^{\prime}=K x / \hbar$, and $\rho(x, 0)$ is defined by $(17)$ and $(8)$.

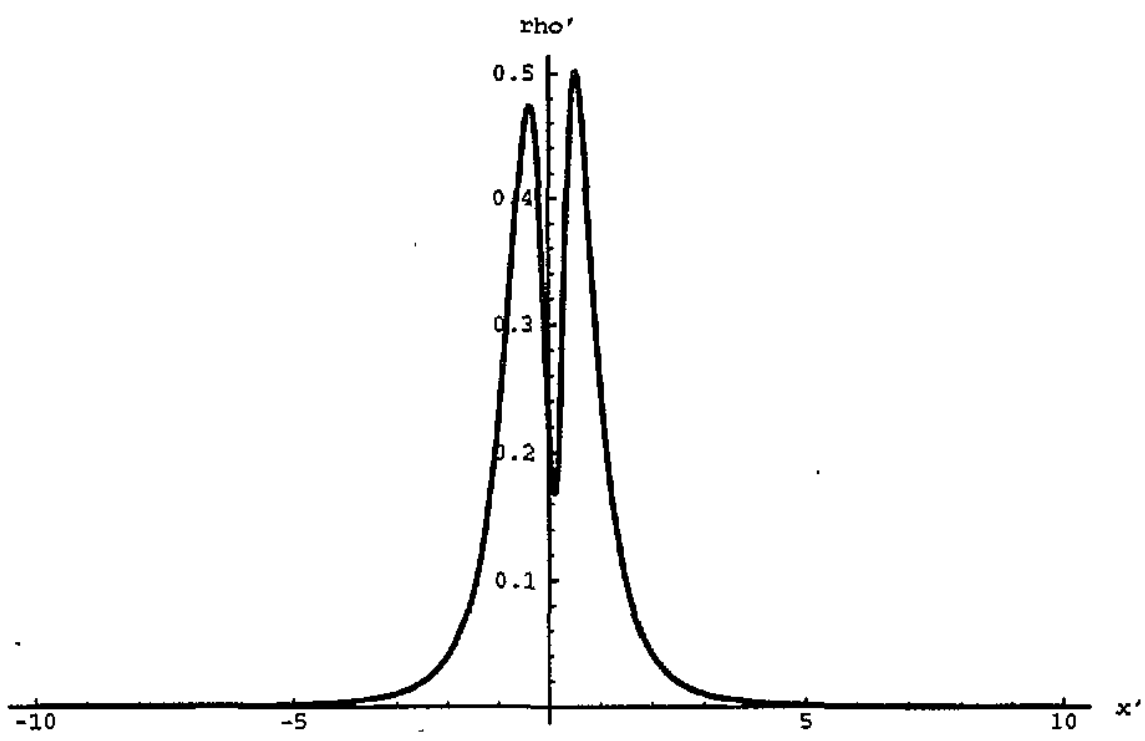

Figure 3. Plot of $\rho^{\prime}\left(x^{\prime}, t^{\prime}\right)$ against $x^{\prime}$ at $t^{\prime}=0.02$. The area under the curve to the left of $x^{\prime}=0$ is greater than that to the right.

In figures 2 and 3 , the probability density $\rho^{\prime}=\hbar \rho / K$ for this case is plotted against $x^{\prime}$ at $t=0$ and $t=0.02 m \hbar / K^{2} \approx 0.94 t_{1}$, respectively, but the backflow effect is so small that it is quite difficult to see from these pictures that the probability has increased on the negative $x$-axis between these times. According to $(17), \rho^{\prime}\left(x^{\prime}, 0\right)$ in figure 2 is an even function of $x^{\prime}$, so the areas under that curve, to the left and right of $x^{\prime}=0$, are equal. On 
the other hand, although the curve in figure 3 is suggestive of a movement of the whole wavepacket to the right, in fact numerical integration shows that the area under this second curve from $x^{\prime}=-7$ to $x^{\prime}=0$ is greater than the area from $x^{\prime}=0$ to $x^{\prime}=7$, by about 0.008 , confirming that an amount of probability equal to about 0.004 has moved from right to the left. This is about one tenth of the maximum amount of probability backflow that can occur in general over any finite time interval, with wavefunctions of the form (11), (12), as will be shown in section 5 .

In this example, it appears from numerical work that it is only during the initial time interval $\left[0, t_{1}\right)$ that probability backflow occurs; for $t>t_{1}$, the probability flux vector $j(0, t)$ is positive (cf figure 1 ) and, although the wavepacket spreads, the probability flows continuously in the positive $x$-direction (see figure 4).

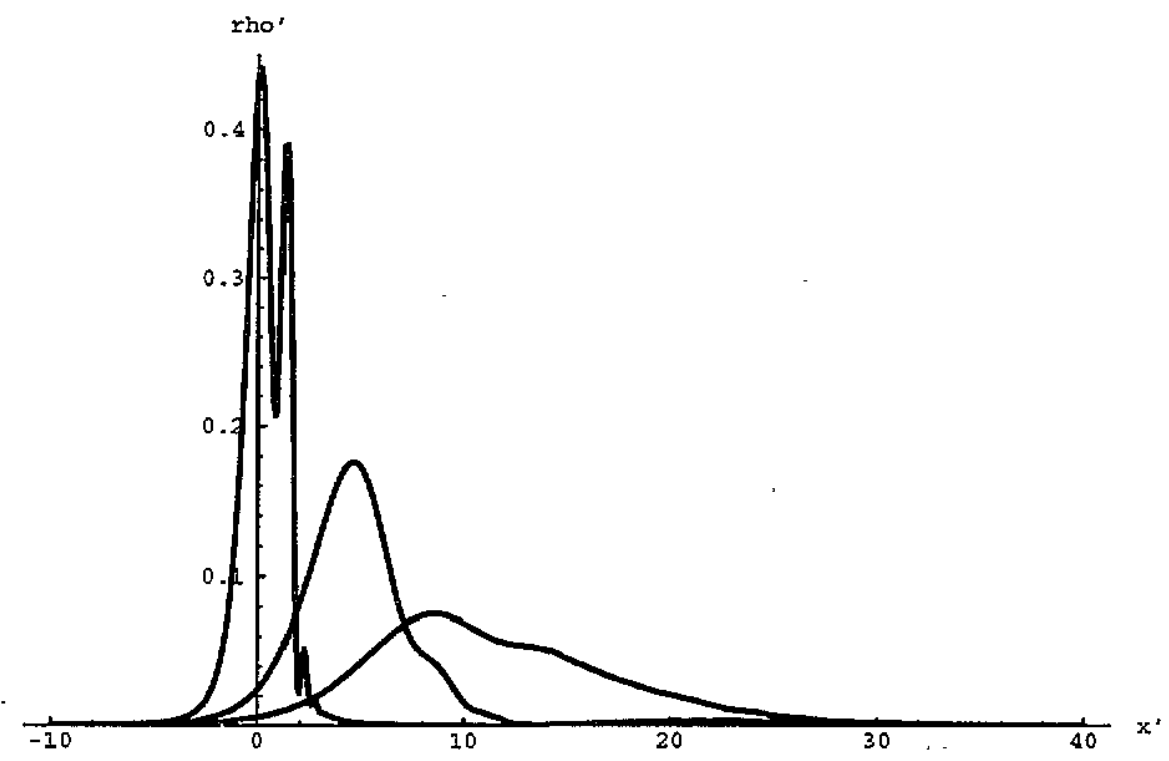

Figure 4. Plot of $\rho^{\prime}\left(x^{\prime}, t^{\prime}\right)$ against $x^{\prime}$ at $t^{\prime}=0.2,4.0$ and 10.0 , showing the movement of the wavepacket and associated,probability mass onto the positive $x^{\prime}$-axis after probability backflow has ceased.

On the other hand, numerical experiments show that in other examples probability backflow can occur on several, and possibly on infinitely many, disjoint subintervals of the time interval $(0, \infty)$. Nevertheless, in every case, an arbitrarily large proper fraction of the total probability mass is located on the positive $x$-axis after a sufficiently long time has elapsed. This is reflected in the general asymptotic result (Whitham 1974)

$\psi(x, t)=\sqrt{\frac{m}{t}} \phi\left(\frac{m x}{t}\right) \mathrm{e}^{\mathrm{i}\left(m x^{2} / 2 \hbar t\right)} \mathrm{e}^{\mathrm{j} \pi / 4}+\mathrm{O}\left(t^{-3 / 2}\right) \quad t \rightarrow \infty, \frac{x}{t}$ fixed

which follows from (11) and which shows that the wavefunction tends to become concentrated on the positive $x$-axis, since $\phi$ vanishes for negative values of its argument. For the example defined by (16), this is illustrated very clearly in figure 5 .

It is worth noting from (16) and (20) that the expectation values $\langle x\rangle,\left\langle v_{\mathrm{op}}\right\rangle$ and $\left\langle H_{\mathrm{op}}\right\rangle$ are finite at all times in this example. Here $H_{\mathrm{op}}=p_{\mathrm{op}}^{2} / 2 m$ represents the total energy 

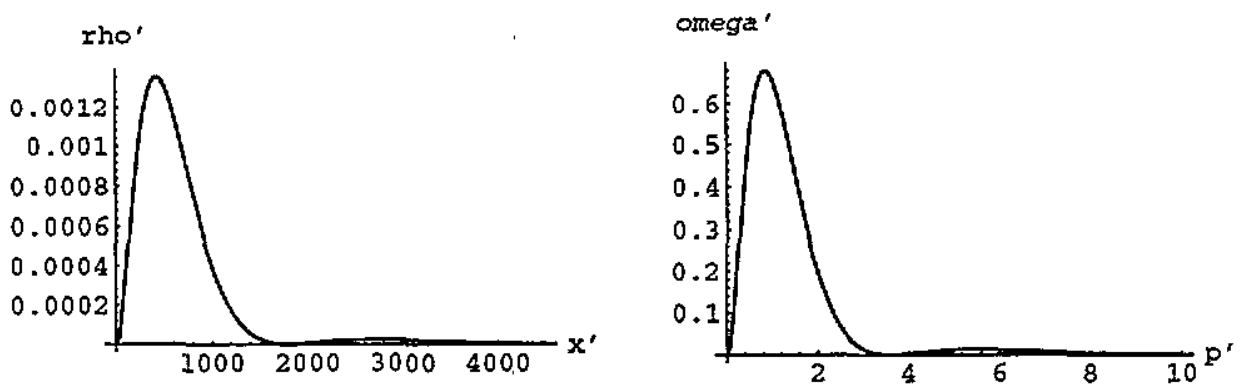

Figure 5. Plots of $\rho^{\prime}\left(x^{\prime}, t^{\prime}\right)$ against $x^{\prime}$ at $t^{\prime}=500$ and $\omega^{\prime}=K\left|\phi\left\langle K p^{\prime}\right\rangle\right|^{2}$ against $p^{\prime}=p / K$, illustrating formula (22) for $\phi$ as in (16). Almost all the probability mass has moved onto the positive $x^{\prime}$-axis at such long times, and the two plots are indistinguishable in shape.

of the system. The dispersions $\Delta x, \Delta v_{\mathrm{op}}$, and $\Delta H_{\mathrm{op}}$ are also finite at all times, where $\Delta x=\left[\left\langle x^{2}\right\rangle-\langle x\rangle^{2}\right]^{1 / 2}$, etc. All of these quantities can be calculated explicitly here; the only non-constant ones are

$\langle x\rangle(t)=t\left\langle v_{\mathrm{op}}\right\rangle(0)=\frac{19 K}{14 m} t \quad \Delta x(t)=\frac{\hbar}{K}\left[\frac{31283}{20580} \frac{K^{4}}{m^{2} \hbar^{2}} t^{2}+\frac{107}{70}\right]^{1 / 2}$.

In particular, $\langle x\rangle$ increases monotonically with $t$ for all $t>0$, even during the interval $\left[0, t_{1}\right)$ when probability backflow is occurring.

\section{Duration and size of the effect}

Once a normalized $\psi(x, t)$ of the form (11) has been found, leading to $j(0, t)<0$ for $t \in[0, T)$ for some $T>0$, then a normalized $\tilde{\psi}(x, t)$ of the same general form can be found for which $\tilde{j}(0, t)<0$ for $t \in\left[0, \lambda^{2} T\right)$, for any $\lambda>0$. To see this, simply replace $\phi(p)$ by

$$
\tilde{\phi}(p)=\sqrt{\lambda} \phi(\lambda p)
$$

in (11), leading to

$$
\tilde{\psi}(x, t)=\frac{1}{\sqrt{\lambda}} \psi\left(\frac{x}{\lambda}, \frac{t}{\lambda^{2}}\right) \quad \tilde{j}(x, t)=\frac{1}{\lambda^{2}} j\left(\frac{x}{\lambda}, \frac{t}{\lambda^{2}}\right) .
$$

It follows that the time interval over which probability flows in the 'wrong' direction can be made arbitrarily long by a suitable choice of wavefunction.

On the other hand, the functions $\psi$ and $\tilde{\psi}$ related as above lead to the same total flow of probability back into $(-\infty, 0)$, over the time intervals $[0, T)$ and $\left[0, \lambda^{2} T\right)$ respectively, because

$$
\int_{0}^{T} j(0, t) \mathrm{d} t=\int_{0}^{\lambda^{2} T} \tilde{j}(0, t) \mathrm{d} t
$$

implying from (7) that

$$
P(T)-P(0)=\tilde{P}\left(\lambda^{2} T\right)-\tilde{P}(0) .
$$


The interesting question, therefore, is not how long this peculiar effect can persist. Rather, it is: over a given time interval $[0, T)$, and allowing all possible choices of $\psi$ of the form (11),(12), what is the greatest increase that can occur in the probability mass on the spatial interval $(-\infty, 0)$ ? More precisely: what is the least upper bound $\vec{\Delta}$ on the set of all possible $\Delta_{P}$ 's that can arise with wavefunctions of this type, where

$$
\Delta_{P}=P(T)-P(0)
$$

in each case?

It follows from the above discussion that $\bar{\Delta}$ is independent of $T$. Furthermore, it is clear that

$$
\bar{\Delta} \leqslant 1
$$

and also that $\bar{\Delta}$ is at least as big as the $\Delta_{P}$ in (21). The main purpose of the next section is to obtain a reasonably accurate estimate of the value of the dimensionless quantity $\bar{\Delta}$.

\section{Probability flow as an eigenvalue problem}

Consider a general $\psi(x, t)$ as in (11), (12). It follows from (9) that

$$
\begin{gathered}
j(0, t)=\frac{1}{4 \pi m \hbar}\left[\int_{0}^{\infty} \mathrm{e}^{\mathrm{i} p^{2} t / 2 m \hbar} \phi^{*}(p) \mathrm{d} p\right]\left[\int_{0}^{\infty} \mathrm{e}^{-\mathrm{i} q^{2} t / 2 m \hbar} q \phi(q) \mathrm{d} q\right]+\mathrm{CC} \\
=\frac{1}{4 \pi m \hbar} \int_{0}^{\infty} \int_{0}^{\infty}(p+q) \mathrm{e}^{\mathrm{i}\left(p^{2}-q^{2}\right) t / 2 m \hbar} \phi^{*}(p) \phi(q) \mathrm{d} p \mathrm{~d} q .
\end{gathered}
$$

According to ( 7$)$, the increase in the probability of finding the particle in the spatial interval $(-\infty, 0)$, over the time interval $[0, T)$, is given by

$$
\Delta_{P}=P(T)-P(0)=-\int_{0}^{T} j(0, t) \mathrm{d} t .
$$

Substitution of (30) in (31), and interchange of the orders of integration, assuming $\phi$ is sufficiently well behaved, gives

$$
\Delta_{P}=\int_{0}^{\infty} \int_{0}^{\infty} \phi^{*}(p) K(p, q) \phi(q) \mathrm{d} p \mathrm{~d} q
$$

after the integration with respect to $t$ is carried out. Here

$$
K(p, q)=\frac{\mathrm{i}}{2 \pi}\left[\frac{\mathrm{e}^{\mathrm{i}\left(p^{2}-q^{2}\right) T / 2 m \hbar}-1}{p-q}\right] .
$$

Note that there is no singularity at $p=q$.

The problem of interest is to maximize $\Delta_{P}$ in (32), subject to the normalization constraint

$$
\int_{0}^{\infty} \phi^{*}(p) \phi(p) \mathrm{d} p=1
$$


and this leads to consideration of the unconstrained maximum of the functional

$$
I(\phi)=\int_{0}^{\infty} \int_{0}^{\infty} \phi^{*}(p) K(p, q) \phi(q) \mathrm{d} p \mathrm{~d} q-\lambda \int_{0}^{\infty} \phi^{*}(p) \phi(p) \mathrm{d} p
$$

where $\lambda$ is a Lagrange multiplier.

At any stationary point of $I$, the Euler-Lagrange equation

$$
\int_{0}^{\infty} K(p, q) \phi(q) \mathrm{d} q=\lambda \phi(p)
$$

must hold, and it then follows from (32) and (34) that, for such a $\phi$,

$$
\Delta_{P}=\lambda
$$

Therefore the problem reduces to that of finding the largest positive eigenvalue, or more precisely, the least upper bound on the spectrum, of the integral operator in (36) with kernel $K(p, q)$.

Note that

$$
K(p, q)=K(q, p)^{*}
$$

so this integral operator is Hermitian, and all its eigenvalues are real. Furthermore, from (37) and the meaning of $\Delta_{P}$, it can be seen that every eigenvalue $\lambda$ must satisfy

$$
-1 \leqslant \lambda \leqslant 1
$$

The eigenvalue equation (36) can be written as

$$
\frac{1}{\pi} \int_{0}^{\infty} \frac{\sin \left[\left(p^{2}-q^{2}\right) T / 4 m \hbar\right]}{(p-q)} \Phi(q) \mathrm{d} q=-\lambda \Phi(p)
$$

where

$$
\Phi(p)=\mathrm{e}^{-\mathrm{i} p^{2} T / 4 m \hbar} \phi(p)
$$

and because (40) is a real equation, it can be assumed without loss of generality that $\Phi$ is real. Furthermore, setting

$$
p=2 \sqrt{\frac{m h}{T}} u \quad q=2 \sqrt{\frac{m h}{T}} v
$$

reduces (40) to

$$
\frac{1}{\pi} \int_{0}^{\infty} \frac{\sin \left(u^{2}-v^{2}\right)}{(u-v)} \varphi(v) \mathrm{d} v=-\lambda \varphi(u)
$$

where

$$
\varphi(u)=\Phi(p) \quad \text { at } p=2 \sqrt{\frac{m h}{T}} u
$$


The form (43) shows that all the eigenvalues $\lambda$, including in particular $\bar{\Delta}$ (the largest positive $\lambda$ ) are independent of $T$ (and, incidentally, of $m$ and $\hbar$ ).

It has not been possible to solve (43) analytically, and numerical methods have been used to estimate $\vec{\Delta}$. With the range of integration in (43) approximated by the interval

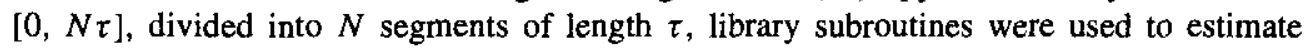
the integral over each subinterval and then to find the largest eigenvalue of the associated discretized eigenvalue problem. With $\tau=0.05$, the estimates obtained were

$$
\begin{array}{ll}
N=100 & \bar{\Delta}=0.0256 \\
N=200 & \bar{\Delta}=0.0297 \\
N=275 & \bar{\Delta}=0.0309 \\
N=500 & \bar{\Delta}=0.0323
\end{array}
$$

which are apparently converging, linearly in $1 / N$ as $N \rightarrow \infty$, to $\bar{\Delta}_{0.05} \approx 0.034$. In the same way, the estimates $\bar{\Delta}_{0.04} \approx 0.035, \bar{\Delta}_{0.025} \approx 0.036, \bar{\Delta}_{0.02} \approx 0.037$ and $\bar{\Delta}_{0.01} \approx 0.038$ were obtained. These in turn are apparently converging, linearly in $\tau$ as $\tau \rightarrow 0$, to the overall estimate

$$
\bar{\Delta} \approx 0.04 \text {. }
$$

(In an independent investigation of (43), our colleague Dr G A Chandler obtained the estimate $\bar{\Delta} \approx 0.038$, and evidence to suggest that the spectrum consists of, in addition, a sequence of smaller positive eigenvalues, with accumulation point at zero, together with the continuum $[0,-1]$. He also obtained an approximate eigenfunction corresponding to the eigenvalue $\bar{\Delta}$. It is hoped that his results will be published separately.)

\section{Flow of negative probability}

The phenomenon can be viewed in an interesting light with the introduction of Wigner's density function (Wigner 1932)

$$
W(x, v, t)=\frac{m}{2 \pi \hbar} \int_{-\infty}^{\infty} \psi^{*}\left(x-\frac{1}{2} y, t\right) \psi\left(x+\frac{1}{2} y, t\right) \mathrm{e}^{-\mathrm{i} m v y / \hbar} \mathrm{d} y .
$$

This function is usually expressed in terms of the canonical pair $(x, p)$ rather than $(x, v)$, but the latter is preferred here because it reflects more clearly the fact that $W$ is the closest analogue which can be constructed in quantum mechanics to the classical density $\mu$ of section 1. For a free particle,

$$
W(x, v, t)=W(x-v t, v, 0)
$$

and $W$ has several properties in common with $\mu(x-x t, v)$. In particular, for wavefunctions of the form (11), which lead to $W$ vanishing for negative values of $v$, the flux vector (9) can be expressed as

$$
j(x, t)=\int_{0}^{\infty} W(x-v t, v, 0) v \mathrm{~d} v .
$$




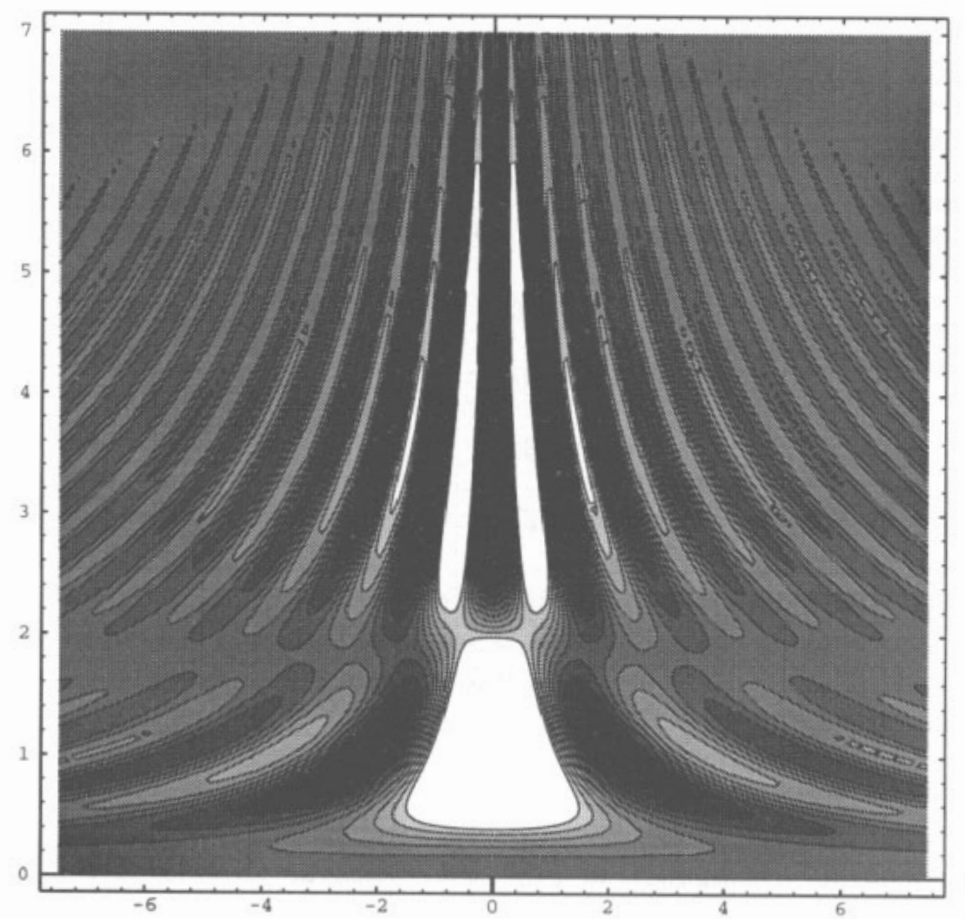

Figure 6. Contour plot of Wigner's density function $W^{\prime}\left(x^{\prime}, v^{\prime}, 0\right)$ for the example defined by (16), with $W^{\prime}=\hbar W / m, v^{\prime}=m v / K$. On darker-shaded regions $W^{\prime}$ is negative, and on lighter-shaded regions it is positive.

This may be compared with (3).

However, it is well known that the $W$, unlike $\mu$, need not be non-negative everywhere, and, in fact, it is at once clear from (49) that if $j(x, t)$ is negative at some $(x, t)$, then $W(x-v t, v, 0)$ must also be negative there, at least for some values of $v$. As an illustration of this, figure 6 shows a contour plot of $W(x, v, 0)$ in the example defined by (16), for which $j(0,0)<0$. Negative values of $W(0, v, 0)$ at some $v$-values can be seen clearly, associated with the darker areas.

Conversely, the appearance of negative values of $j$, despite the restriction of the integral in (49) to positive values of $v$, can be regarded as a direct consequence of the fact that Wigner's function can take negative values.

The interpretation of negative values of $W$ and, more generally, the difficulties of defining positive semi-definite phase-space density functions in quantum mechanics have been much discussed (Wigner 1932, Dirac 1945, Moyal 1949, Barut 1957), but Feynman (1987) has argued that interpreting negative values of Wigner's density function in the obvious way, in terms of negative probabilities, need not lead to unphysical conclusions. (In this connection, see also Dirac (1942) and Bartlett (1945).) Observables correspond to functions $f(x, v)$ which have just the structure necessary to avoid such conclusions, when their mean values are calculated using $W$ as probability density. For descriptive purposes then, $W(x, v, t) \delta x \delta v$ can be interpreted as the probability that the particle 'has' a coordinate within $\delta x$ of $x$, and a velocity within $\delta v$ of $v$ at time $t$, even though this probability can be 
negative; but it must be kept in mind that there is no observable $f(x, v)$ which has $W \delta x \delta v$ as its expectation value, and that the indeterminacy principle precludes the observation of an event $(x, v)$.

Whatever the difficulties of such an interpretation, it does provide a very simple way of describing the peculiar quantum effect which has been the subject of this paper. According to (49), all the probability flows in the direction of the particle's velocity. However, not all the probability is positive, and a flow of negative probability in the positive $x$-direction is mathematically equivalent to a flow of positive probability in the negative $x$-direction; it is evidently just such a (net) flow of negative probability in the direction of the particle's velocity that gives rise to the effect in question. Figure 7 shows, at a later time, a contour plot of the Wigner function of figure 6; all regions of the plot have moved to the right in accordance with (48). Whenever the darker (negative) regions passing across the line $x=0$ outweigh the lighter (positive) regions, then there is a net flow of positive probability to the left over that line at that time.

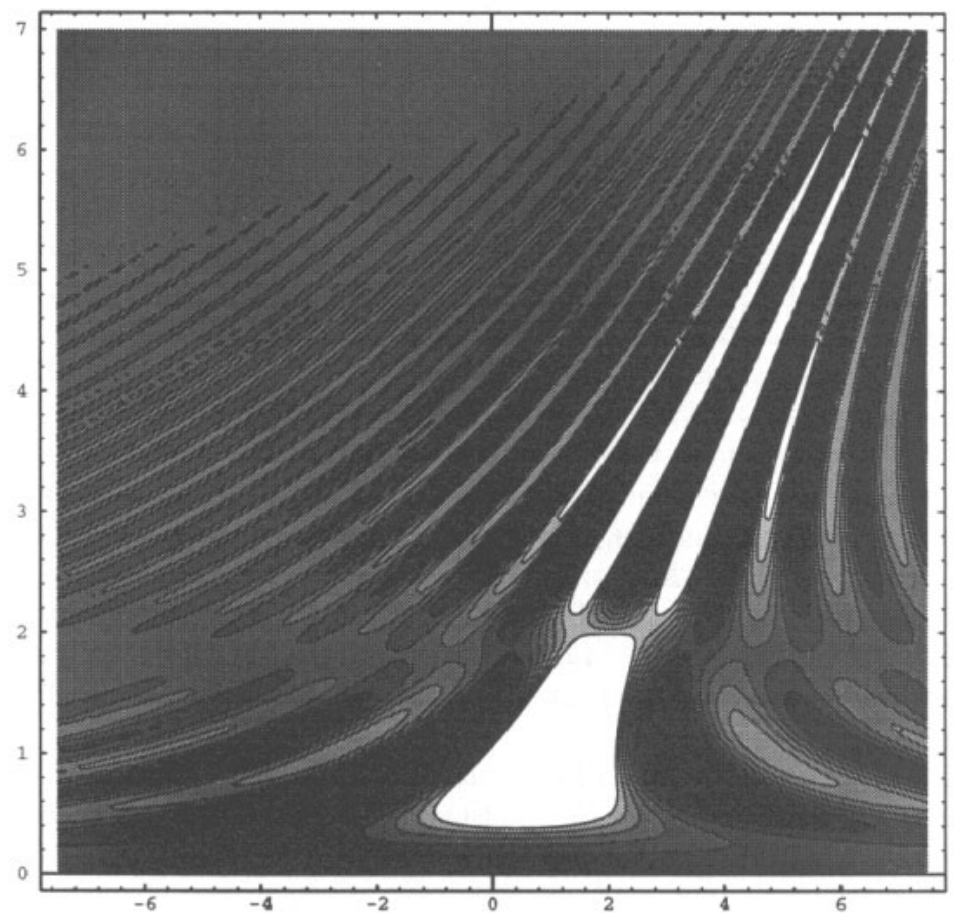

Figure 7. Contour plot of $W^{\prime}\left(x^{\prime}-v^{\prime} t^{\prime}, v^{\prime}, 0\right)$ at $t^{\prime}=1$, illustrating the way in which $W^{\prime}$ evolves in time.

Experimental observation of this remarkable effect is possible in principle, as suggested in the introduction. The main difficulty would seem to be preparation of initial states of the required type. If that could be achieved, in a reproducible way, then on a large ensemble of replicas of the one-particle system, all prepared in the same state, measurements of the velocity (or momentum) could be made on one subset to confirm that the wavefunction contains only positive velocity (momentum) components, and measurements of the position 
could be made after various elapsed times on a second disjoint subset, in order to confirm that the probability of finding the particle 'upstream' of a given point was in fact increasing. Such a procedure seems unlikely to be realizable. A more practicable alternative might be, for a particle with an electric charge, to prepare various initial states which all contain only positive velocity components but are otherwise arbitrary, and in each case to measure at some subsequent time the electric current density vector. Since this is proportional to the probability flux vector, the observation in any one instance of a current density directed opposite to the velocity (muliplied by the charge) would confirm the existence of the effect in question. In the light of the comments made above, any such successful observation could be regarded as providing direct evidence of negative values of Wigner's function, if not, indeed, of negative probabilities.

\section{Concluding remarks}

Even when wave-particle duality is borne in mind, it is quite counter-intuitive that there exist quantum states of a free particle, with positive $x$-component of velocity, and with increasing probability of finding the particle in the spatial interval $\left(-\infty, x_{0}\right)$, for any given $x_{0}$. The greatest amount of probability backflow that can occur over any given time interval $[0, T)$ has been found to be $\bar{\Delta} \approx 0.04$, and the appearance of this dimensionless quantity $\bar{\Delta}$ in the quantum mechanics of a free particle is itself remarkable, because it is not possible to construct such a quantity from the parameters $m$ and $\hbar$ appearing in Schrödinger's equation (10), nor even from $m, \hbar$ and $T$. It is the fact that the wavefunction $\psi$ itself has dimensions of [length] ${ }^{-1 / 2}$ which enables the construction of dimensionless quantities like $P(t)$ in (5) and then, indirectly, $\bar{\Delta}$.

It could be said that $\bar{\Delta}$ in some way characterizes a free particle in quantum mechanics, but, since it is independent not only of the length $T$ of the time interval over which the backflow occurs but also of the mass $m$ of the particle and of $\hbar$, it seems more appropriate to say that $\bar{\Delta}$ is characteristic of the very nature of the quantum mechanical description itself, in terms of the complex wave equation (10). In this sense, and despite its lack of dependence on the value of Planck's constant, it is truly a 'quantum' number. This is true also of any other eigenvalues in (36), whose meaning is more obscure. The existence of a quantum effect with no classical analogue, but with a magnitude that is independent of Planck's constant, emphasizes the subtlety of the relationship between a quantum mechanical system and its classical counterpart; it is often implied that the latter can be obtained from the former simply by 'letting $\hbar \rightarrow 0$ '.

Instead of a free particle, one that is subject to a force in the positive $x$-direction could be considered. At least in the case of a constant force $F$, it is then easy to show that, just as for a free particle, a state that contains only positive momentum components initially will do so at all subsequent times. Again, situations exist where probability backflow occurs over a time interval of length $T$, no matter what the size of $F$. An example is provided by again taking the initial value of the wavefunction to be defined by (16). In such cases the maximum amount of probability backflow that can occur is expected to depend on the values of $F, \hat{h}, T$ and the mass $m$ of the particle, through the dimensionless combination $F^{2} T^{3} / m \hbar$.

It would also be possible to consider a single particle in relativistic quantum mechanics, for example a Dirac electron. Here the relationship between the observable velocity and the momentum is more contentious. States could be constructed that contain only positive $x$-components of momentum, but again lead to a flow of probability in the negative $x$ direction over a time interval of length $T$. In this case, it is expected that the maximum 
probability backflow will be found to depend on the dimensionless combination $m c^{2} T / \hbar$, where $c$ is the speed of light.

\section{Acknowledgments}

It is a pleasure to thank members of the Department of Mathematics, in particular Dr G A Chandler, for several helpful discussions.

\section{References}

Allcock G R 1969 Ann. Phys., NY 53 311-48

Bartlett M S 1945 Proc. Camb. Phil. Soc. 41 71-5

Barut A O 1957 Phys. Rev. 108 565-9

Belinfante F J 1973 A Survey of Hidden-Variables Theories (Oxford: Pergamon) pp 105-6

Bohm D 1952 Phys. Rev. 85 166-179, 180-193

Broglie L de 1926 C. R. Acad. Sci. Paris 183 447-8

- 1960 Nonlinear Wave Mechanics: A Causal Interpretation transi A J Knodel and J C Miller (Amsterdam: Elsevier) pp 136-9

Dirac P A M 1942 Proc. R. Soc. A $1801-40$

1945 Rev. Mod. Phys. 17 195-9

Feynman R P 1987 Quantum Implications ed B J Hiley and F D Peat (New York: Routledge and Kegan Paul) pp 235-48

Moyal J E 1949 Proc. Camb. Phil. Soc. 45 99-124

Whitham G B 1974 Linear and Nonlinear Waves (New York: Wiley) pp 373-7

Wigner E P 1932 Phys. Rev. 40 749-59 